\title{
Coke Ratio Prediction Based on Immune Particle Swarm Neural Networks
}

\author{
Yang Kai $^{1,2, *}$, Jin Yonglong ${ }^{3}$ and He Zhijun ${ }^{1}$
}

${ }^{1}$ School of materials and metallurgy, University of Science and Technology LiaoNing, LiaoNing 114051, China; ${ }^{2}$ School of Software, University of Science and Technology LiaoNing, LiaoNing 114051, China; ${ }^{3}$ Technical Center of TangShan Iron \& Steel, HeBei Iron \& Steel Group, HeBei, 063016, China

\begin{abstract}
The clonal selection mechanism and vaccination strategy of immune system are introduced into particle swarm optimization algorithm in this paper, in order to enhance the ability of global exploration of PSO, avoiding getting into local optimum and improving the accuracy and convergence speed of BP networks. The global Cauchy mutation operator and local Gauss mutation operator are used to improve the ability of searching global optimization and the accuracy of local optimization. Then the weights and thresholds of neural networks are trained by applying the immune particle swarm optimization. Finally the coke ratio forecasting model is established based on the modified BP neural networks optimized by immune particle swarm optimizer. The result shows the forecast accuracy is more accurate than both the BP neural networks optimized by the standard PSO and the traditional BP neural networks, and provides an effective way to reduce the coke ratio and achieve energy conservation and emission reduction for iron and steel enterprise.
\end{abstract}

Keywords: Coke ratio, immune, neural networks, particle swarm optimizer.

\section{INTRODUCTION}

Coke ratio is one of the most important criteria in blast furnace iron-making and makes a strong impact on energy consumption. There is a pressing need to reduce fuel consumption for energy conservation, emission reduction, cost reduction and environmental protection. The factors related with coke ratio involved various aspects in the blast furnace. Factors influencing the coke ratio are multitudinous with strong cross-coupling and the inner rules are very difficult to master. Therefore, the relation between coke ratio and other parameters has strong nonlinear characteristics. The traditional prediction methods based on the experience or mathematical model would often not achieve anticipated performance. In recent years, applying the modern intelligence computing methods to engineering areas is a new research interest in prediction technology.

Fan et al. [1] use the improved neural networks to predict the coke ratio in blast furnace. In [2], Zhou et al. combine the cluster analysis and neural networks model to predict the coke ratio; the results show the new prediction model can enhance the accuracy of the algorithm. Han et al. [3] apply the genetic algorithm to optimize the initial weights of neural networks and then establish forecast model for coke ratio. In [4], particle swarm optimization combined with chaotic local search is used to optimize the initial connection weights of the BP neural network model. Chen et al. [5] use generalized regression neural network to set up the neural network models of iron-making process. In [6], the particle swarm optimization is improved by immunity algorithm through using

*Address correspondence to this author at the School of Software, University of Science and Technology LiaoNing, Anshan, Liaoning, 114051, P.R. China; E-mail: asyangkai@126.com memory, density mechanism, vaccination and immune selection of IA. Li [7] propose an immune binary particle swarm optimization method to learn Bayesian networks structures. In [8], Sun et al. adopt clonal algorithm combined with the Gauss variation and Cauchy variation to optimize parameters of structure-changed fuzzy neural networks.

This paper lead the clonal selection mechanism and vaccination strategy into particle swarm optimization, set up a predictive model for coke ratio based on immune particle swarm neural networks (CSVS-PSO-BP). The global Cauchy mutation operator and local Gauss mutation operator are used in antibody variation process to improve the ability of searching global optimization and the accuracy of local optimization. Experimental results show that the proposed approach outperforms other compared algorithms on the coke ratio predictive problems.

\section{PSO ALGORITHM}

In PSO, a particle $x_{i}=x_{i 1}, x_{i 2}, \cdots, x_{i D}$ represents a potential solution to a problem in a $D$-dimensional search space. The fitness function predefined decides the merits of a particle. The velocity for particle $i$ is represented as $v_{i}=v_{i 1}, v_{i 2}, \cdots, v_{i D}$. Every particle keeps a record of the best position that it has ever visited. Such a record is called the particle's previous best position and denoted by $p_{i}=p_{i 1}, p_{i 2}, \cdots, p_{i D}$. The best particle in the swarm is represented by $p_{g}=p_{g 1}, p_{g 2}, \cdots, p_{g D}$.

In every iteration, the position and velocity of each particle are updated by following two formulas: 
$v_{i d}^{k+1}=w v_{i d}^{k}+c_{1} r_{1}\left(p_{i d}-x_{i d}^{k}\right)+c_{2} r_{2}\left(p_{g d}-x_{i d}^{k}\right)$

$x_{i d}^{k+1}=x_{i d}^{k}+v_{i d}^{k+1}$

where $i=1,2, \cdots, M ; d=1,2, \cdots, N$, and $M$ is the size of the swarm; $k$ determines the iteration number; $c_{1}, c_{2}$ are two positive constants, called cognitive and social parameter respectively; $r_{1}, r_{2}$ are two random functions in the range $[0,1] . w$ is called inertia weight which coordinate the global and local searching ability of PSO algorithm. Normally, $w$ is reduce gradually with the increase of iteration number.

\section{THE IMPROVEMENT THOUGHT OF PSO}

\subsection{Affinity Calculation}

In immune particle swarm algorithm, a particle is thought as antibody, and the objective function is regarded as antigen. In iterative process of the algorithm, the clonal selection and mutation of an antibody are carried according to the affinity between antibody and antigen, so as to enhance the global exploration ability of the algorithm. Calculation affinity is the backbone of the whole algorithm. The error function in predicting model is regularly defined as an objective function. The affinity of a particle $i$ is defined as $: a f f_{i}=1 / E_{i}$. In the improved algorithm, the swarm is divided into two sub-swarm by means of average affinity of the whole population.

\subsection{Clone the Antibodies}

The clonal mechanism of antibody in immune algorithm is used in CSVS-PSO. The antibodies with high concentration and low affinity are inhibited. Conversely, the antibodies with low concentration and high affinity are promoted. In this paper, the two indexes are taken into account to select antibodies. The selective probability $p_{i}$ of an antibody consists of two parts [6]: $p_{i}^{1}$ based on concentration and $p_{i}^{2}$ based on affinity. We have:

$$
p_{i}=\text { á } p_{i}^{1}+(1-\alpha) p_{i}^{2},
$$

where

$$
p_{i}^{1}=\frac{a f f_{i}}{\sum_{n=1}^{M} a f f_{i}}
$$

The antibody concentration is defined as :

$$
\text { Diversity }_{i}=\frac{1}{\sum_{n=1}^{M} \mid \text { Fitness }_{i}-\text { Fitness }_{n} \mid}
$$

hence,

$$
p_{i}^{2}=\frac{\text { Diversity }_{i}^{-1}}{\sum_{n=1}^{M} \text { Diversity }_{n}^{-1}}
$$

Half of particles in each sub-swarm are selected in CSVSPSO to be cloned according to the selective probability $p_{i}$.

\subsection{Mutation}

The idea of immune clonal algorithm is that in each evolution, some clonal antibodies are generated around candidate solutions according to affinity and concentration, then carry out antibody variation to expand search range of solutions. Two mutation operators are adopted in this paper [8]: global Cauchy mutation operator and local Gauss mutation operator. The global Cauchy mutation operator can search the global optimal solution on a large scale in solution space to get better global search ability. The local Gauss mutation operator can search the global optimal solution with high accuracy to enhance local search ability. The mixed mutation operators can increase search space as well as enhance the local search capability.

\subsubsection{Local Gauss Mutation}

The local Gauss mutation is carried out for the antibody whose affinity is above the average affinity of a swarm so as to perform accurate search around the excellent solution. The formula is as follows:

$$
\begin{aligned}
& x_{i}^{\prime}=x_{i}(j)+\sigma_{i}^{\prime}(j) N_{j}(0,1) \\
& \sigma_{i}^{\prime}(j)=\sigma_{i}(j) \exp \left(\tau^{\prime} N(0,1)+\tau N_{j}(0,1)\right) \\
& j=1,2, \cdots, n, i=1,2, \cdots, \text { Size }
\end{aligned}
$$

\subsubsection{Global Cauchy Mutation}

The global Cauchy mutation is carried out for the antibody whose affinity is below average affinity of swarm so as to enlarge search space through increasing varying scope of position and effectively prevent local optimization. The formula is as follows:

$$
\begin{aligned}
& x_{i}^{\prime}=x_{i}(j)+\sigma_{i}^{\prime}(j) C_{j}(0,1) \\
& \sigma_{i}^{\prime}(j)=\sigma_{i}(j) \exp \left(\tau^{\prime} N(0,1)+\tau N_{j}(0,1)\right) \\
& \mathrm{j}=1,2, \cdots, \mathrm{n} \quad \mathrm{i}=1,2, \cdots, \text { Size }
\end{aligned}
$$

$N(0,1)$ denotes a normally distributed one-dimensional random number with mean zero and standard deviation one. $N_{j}(0,1)$ indicates that the random number is generated anew for each value of $j . C_{j}(0,1)$ denotes a Cauchy random number for each value of $j$ centered at zero with a scale parameter of 1 . The factors $\tau=\frac{1}{\sqrt{2 \sqrt{n}}}, \tau^{\prime}=\frac{1}{2 \sqrt{n}}$.

After the two mutation processes are implemented in two sub-swarms respectively, we select twenty percent of excellent particles in the result set according to the affinity of antibodies and replace ten percent of inferior particles in the original swarm. The essence of the hybrid mutation is to generate a new particle swarm near the promising candidate solution such that the search space is enlarged and the diversity of swarm is enhanced to be trapped in local minima. In the meantime, the performance of the improved algorithm could be enhanced rapidly. 


\subsection{Vaccination Strategy and Immune Selection}

Given an antibody, a vaccination means modifying the genes on a number of bits in harmony with priory knowledge so as to gain better affinity value [9]. In the immune particle swarm algorithm, we make the global optimal solution to be the vaccine and the individual with the lowest affinity in swarm as the antibody to be vaccinated.

Suppose the size of genes in vaccine is $n$, a string including characters 0 and 1 with length $n$ would be generated randomly. If a bit of the string is 1 , it means the same bit of the antibody would be vaccinated. Otherwise, the gene-bit remain unchanged.

If the affinity of the antibody vaccinated is smaller than that of the parent, which means degeneration has happened in the evolution process, the parent would replace the current individual in the next generation.

\section{THE IMMUNE PARTICLE SWARM OPTIMIZER}

This paper introduces the clonal selection and vaccination strategy in artificial immune system to standard particle swarm optimization algorithm, and proposes an improved particle swarm optimization based on immune mechanism(CSVS-PSO). The flow diagram of CSVS-PSO is presented in Fig. (1). We can see from Fig. (1) that the algorithm initializes the population firstly, then calculates the fitness of each particle to obtain the pbest and gbest based on this. Subsequently, the next generation population is generated on which the immune clonal selection and vaccination strategy will be carried out. In the first dotted box, the immune clonal selection is inside. All the particles are viewed as antibodies, compute affinity of the antibodies and divide the population into two sub-swarms, clone antibodies according to affinity and concentration and perform two kinds of mutation respectively, finally choose some excellent antibodies to replace the original inferior ones to improve the performance of algorithm. In the second dotted box, part of vectors in global optimum are used as vaccine to plug into the antibodies with low affinity, and immune selection is implemented afterward.

The new algorithm can be summarized as follows:

(1) Initialize position and velocity of all the particles randomly in the $\mathrm{N}$ dimension space.

(2) Calculate the fitness of all particles and obtain the global the global optimum position Gbest and the individual optimum position Pbest.

(3) Generate the next generation $X C$ according to the formulas (1) and (2).

(4) Divide the swarm $X C$ into two sub-swarms $X_{m}$ with higher affinity and $X_{r}$ with lower affinity by means of average affinity of the whole population.

(5) Calculate the selection probability of each particle according to both affinity and concentration, retain the better half particles $X_{m h}$ and $X_{r h}$.

(6) Perform Local Gauss mutation to $X_{m h}$ and Global Cauchy mutation to $X_{r h}$.
(7) Merge the mutation results and select twenty percent of excellent particles in result sets to replace ten percent of inferior particles in the original swarm.

(8) Select part of vectors in global optimum as vaccine randomly to plug into the antibodies with low affinity.

(9) Immune selection: If the affinity of the antibody vaccinated is smaller than that of the parent, the parent would replace the current individual in the next generation.

(10) Calculate the fitness of population and update Gbest and Pbest.

(11) Repeat Steps 3 - 10 until a stop criterion is satisfied or a predefined number of iterations are completed.

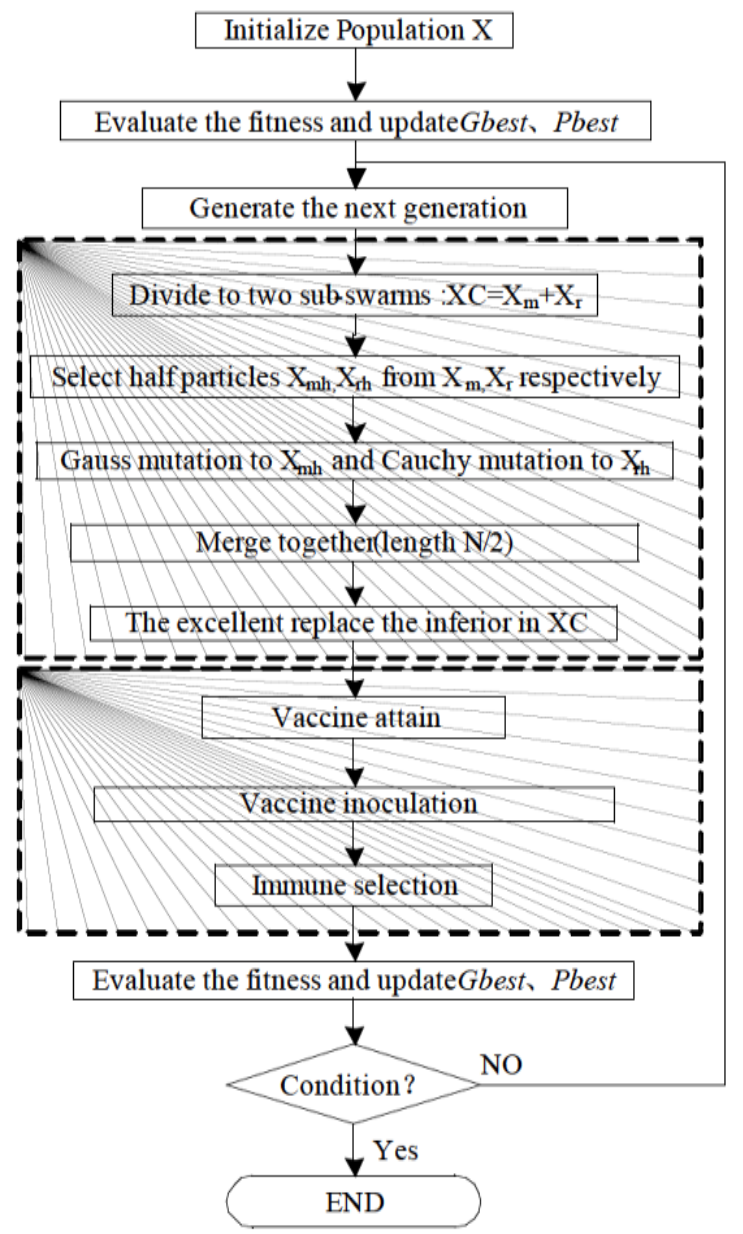

Fig. (1). The flow diagram of the algorithm.

\section{PREDICTION ALGORITHM BASED ON CSVS-PSO AND BP}

\subsection{Neural Network Training}

We use the improved PSO based on immune clonal selection strategy to optimize the weights and threshold of BP neural networks. When the neural works is trained, the connection weights between neurons and threshold of BP neural networks are encoded to real number string shown as a particle, $x_{i}=\left(x_{i 1}, x_{i 2}, \cdots, x_{i L}\right), i=1,2, \cdots, N$, where 


$$
L=R S+S T+S+T
$$

$R, S$ and $T$ represent the number of input layer, the number of hidden layer and the number of output layer.

In the executive procedure of algorithm, each particle is decomposed as weights and threshold of neural works, and the fitness of particles is calculated constantly until a stop criterion is satisfied or a predefined number of iterations are completed. The optimal particle is the training result of weights and threshold in neural works.

The fitness of individual in population is defined as mean square error of the sample data:

$$
\text { fitness }_{i}=\frac{\sum_{j=1}^{M}\left(y_{j-} O_{j}\right)^{2}}{M}
$$

where $\mathrm{M}$ is the number of test sample, $y_{j}$ is predictive value of the i-th sample, $O_{j}$ is observed value of the i-th sample.

\subsection{The Input Parameters}

The coke ratio is affected by many factors with strong cross-coupling. It is difficult to clearly define some mecha-
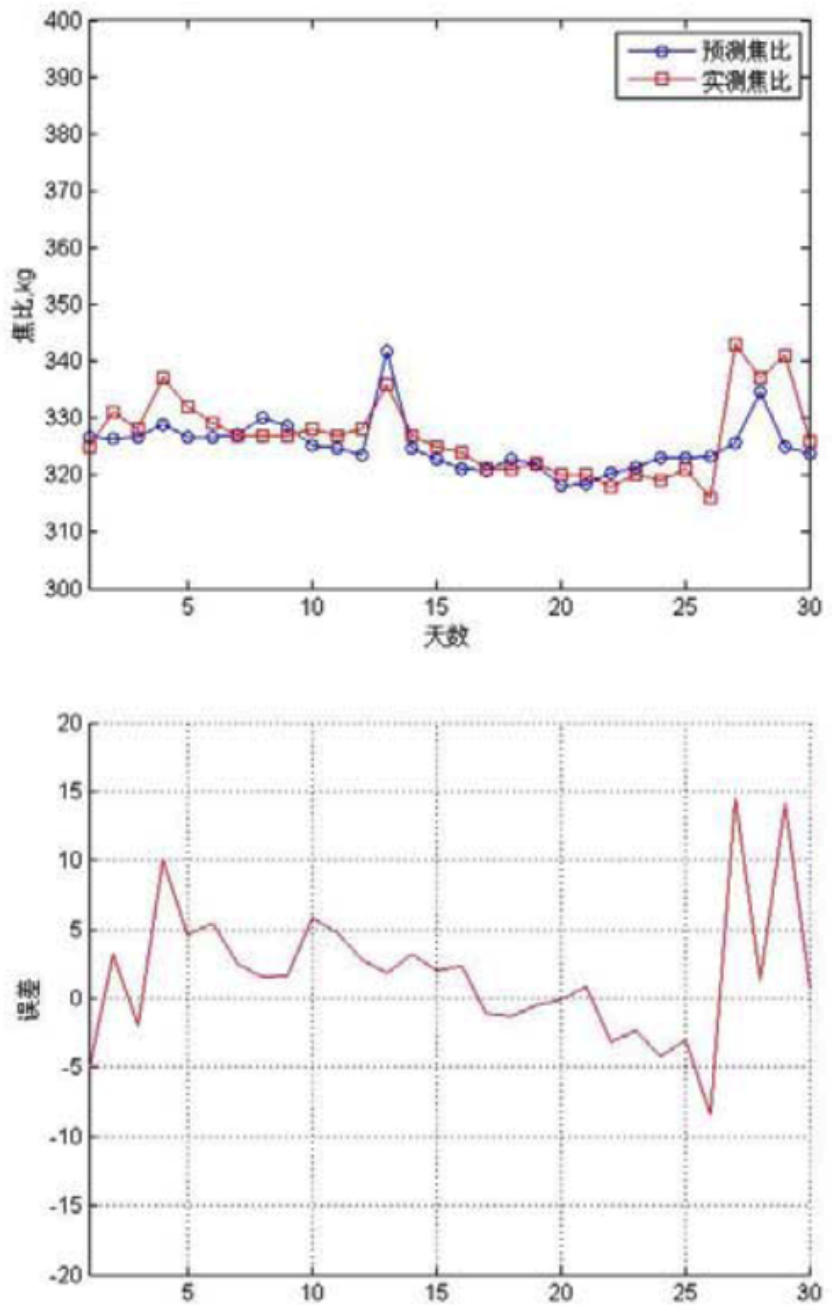

Fig. (2). The predictive result of CSVS-PSO-BP. nism and numerical relationship among those arguments. This make the relationship between coke ratio and other related arguments have strong nonlinear characteristics. In the predictive model, we carefully select nine arguments as input values of neural works combined with data captured in the field and the associated degree of coke ratio. The selected arguments are as follows: coal ratio, air volume, rate of oxygen, hot blast temperature, permeability-index, peak Temperature, grade of feeding into furnace, silicon content, sulfur content. The coke ratio is taken as the output of neural networks.

\section{EXPERIMENTAL RESULTS}

The iron-making data in simulation come from the blast furnace with $3200 \mathrm{~m}^{3}$ in a large steel enterprise from April 2012 to September 2012. There are 181 samples in all. The preceding 5 months with 151 samples is used as training samples and the data in September is used as test samples. Because the differences of value exist considerably between various input arguments of neural works, we use normalized treatment to the input variables and the output variables. The network structure is 9-9-1. The initial range of weights and threshold is $[-1,1]$. In order to test the performance of our
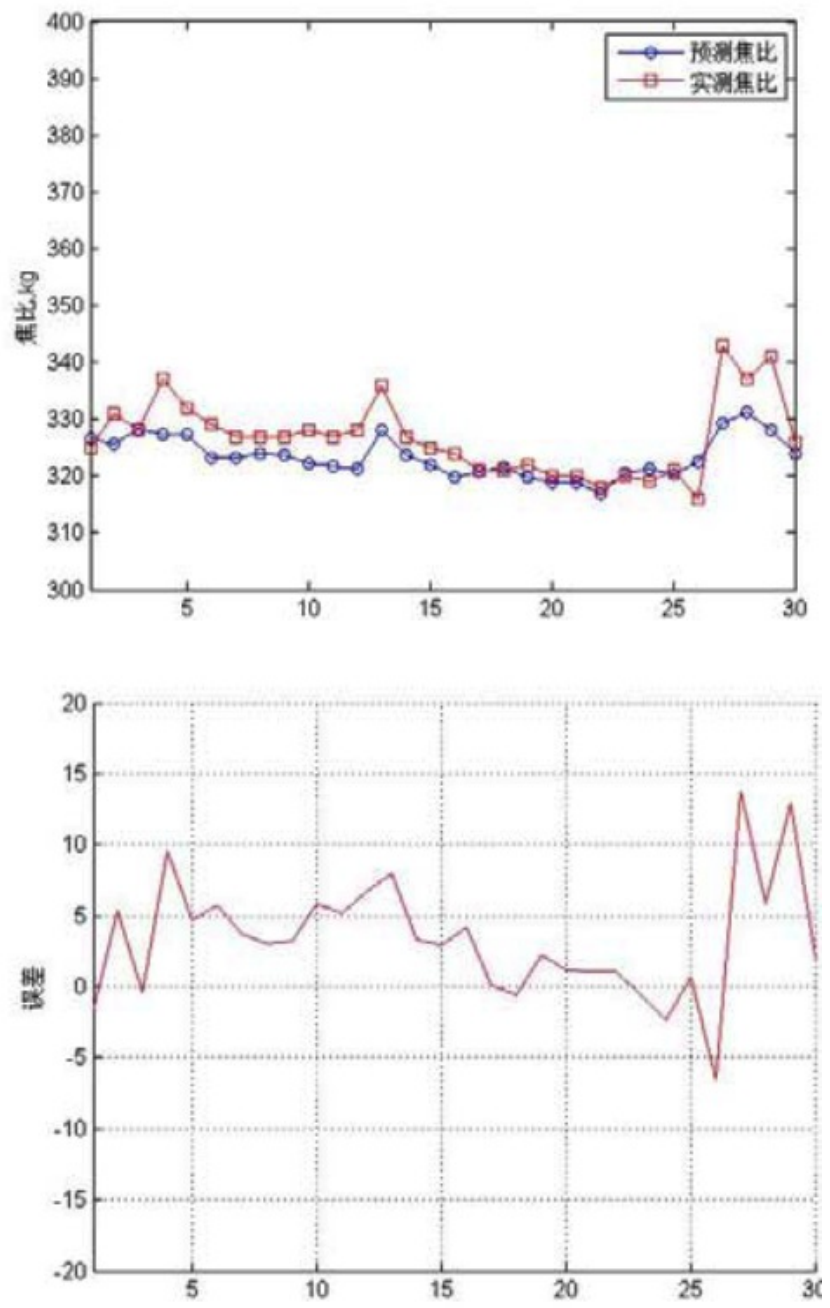

Fig. (3). The predictive result of PSO-BP. 
Table 1. Experimental results for different algorithms.

\begin{tabular}{|c|c|c|c|}
\hline & Mean Absolute Percentage Error & Mean Absolute Difference & Mean Square Error \\
\cline { 2 - 4 } & MAPE & MAD & 27.2793 \\
\hline \hline CSVS-PSO-BP & 0.0116 & 3.8167 & 28.9034 \\
\hline PSO-BP & 0.0125 & 4.1423 & 42.2701 \\
\hline BP & 0.0169 & 5.5494 & \\
\hline
\end{tabular}
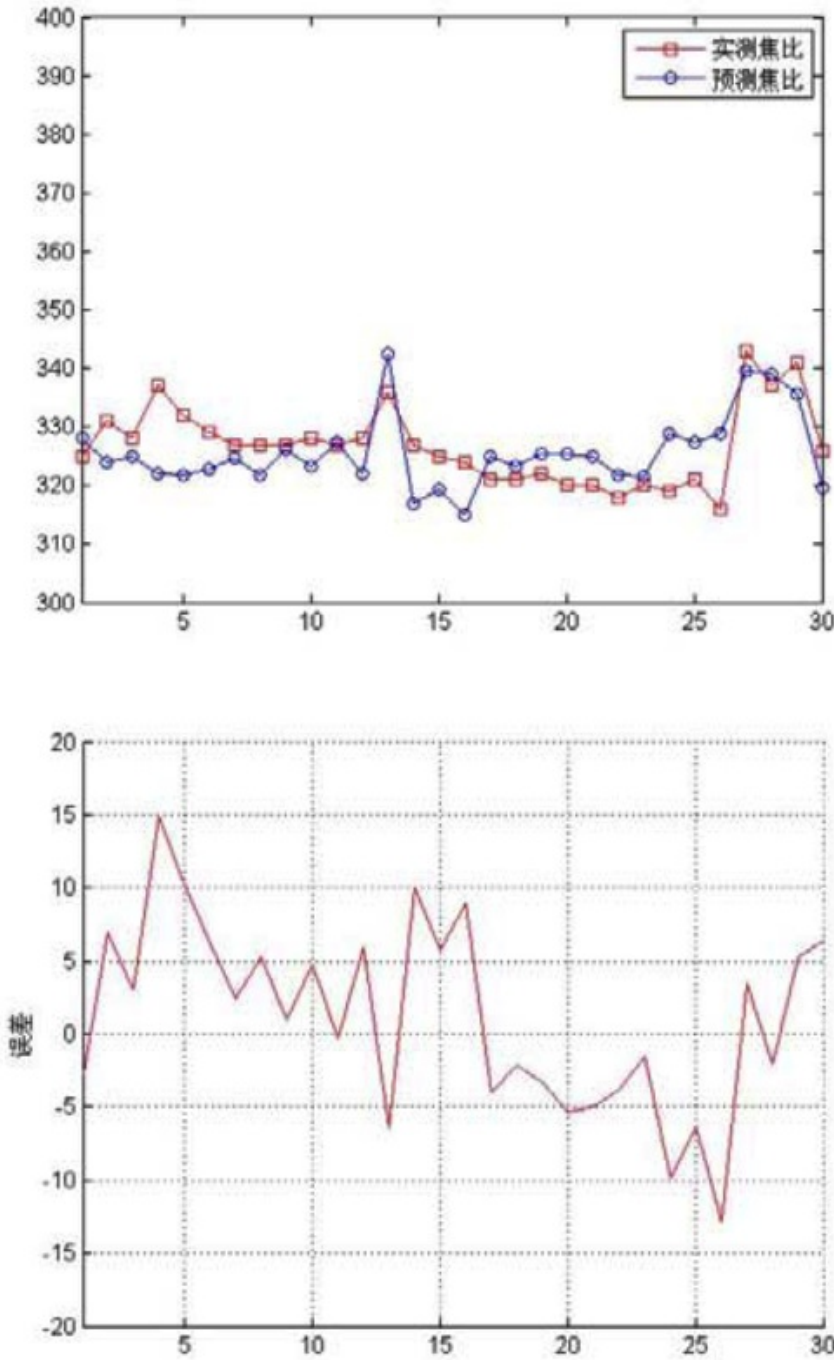

Fig. (4). The predictive result of BP.

proposed algorithm, the final results of the new model are compared with PSO-BP algorithm and BP algorithm. In training process of CSVS-PSO-BP and PSO-BP, the initial arguments are: $w_{\max }=0.9, w_{\min }=0.3, c_{1}=2.5, c_{2}=1.5$, the population size $\mathrm{N}=60$, and the max iteration number 400 .

The predictive results on the given dataset of CSVSPSO-BP, PSO-BP and BP are shown in Figs. (2-4). The Mean Absolute Percentage Error (MAPE), Mean Absolute Difference (MAD), and Mean Square Error (MSE) of the three predictive models are shown in Table 1. From Figs. (2-
4), it can be seen that there is better fitting efficiency for the CSVS-PSO-BP than for the PSO-BP and BP. The results in Table 1 show that the improved BP based on immune particle swarm optimization has more predictive precision with MAPE i.e. only 1.16 percent than PSO-BP and BP algorithms with MAPE percent 1.25 and percent 1.69 respective1y. The results of simulation in the CSVS-PSO-BP predictive model agree well with the experimental data. The model can meet the demands of actual problem and are appropriately applied to predict the coke ratio in furnace.

\section{CONCLUSION}

This paper introduces a new form of the particle swarm optimizer through introducing the clonal selection mechanism and vaccination strategy, uses it to optimize the performance of BP neural works, finally, sets up a predictive model based on CSVS-PSO and BP algorithm for coke ratio. The results obtained show that CSVS-PSO-BP approach gives a better forecast in terms of accuracy for coke prediction in furnace. These works give a method for an in-depth study of energy saving and consumption reducing in producing process for furnace.

\section{CONFLICT OF INTEREST}

The authors confirm that this article content has no conflict of interest.

\section{ACKNOWLEDGEMENTS}

This work was financially supported by scientific research fund of Liaoning provincial education department (L2014118) and the Anshan science and technology park.

\section{REFERENCES}

[1] Z. Fan, G. Oiu, J. Jia, and C. Bai, "Method to predict the coke rate based on BP neural network," Journal of Chongging University, NaturaI Science Edition, vol. 25, no. 6, pp. 85-87, 91, 2002.

[2] Y. Zhou, W. Yu, X. Dong, and J. Dong, "A prediction model for blast furnace coke ratio with clustering analysis and neural network," Journal of University of Science and Technology Liaoning, vol. 33, no. 3, pp. 245-247, 2010.

[3] H. Han, and X. Yan, "The model for predicting the coke rate based on genetic algorithm and artificial neural network," Journal of Hebei Institute of Technology, vol. 28, no. 2, pp. 23-27, 2006.

[4] X. Chen, W. Weng, M. Wu, and W. Cao, "BP neural network model of coke consumption of sintering process based on chaotic PSO algorithm," Computers and Applied Chemistry, vol. 30, no. 10, pp. 1223- 1226, 2013

[5] C. G. L. Wentao, "Application of generalized regression neural network in BF iron- making energy intensity prediction," Energy for Metallurgical Industry, vol. 32, no.4, pp. 15-18, 2013. 
[6] J. Wu, H. Zuo, and Y. Chen, "A combined forecasting method based on particle swarm optimization with immunity algorithms," Systems Engineering-Theory Methodology Applications, vol. 15, no. 3, pp. 229-233, 2006.

[7] X. L. Li, "A particle swarm optimization and immune theory-based algorithm for structure learning of Bayesian networks," International Journal of Database Theory and Application, vol. 3, no. 2, pp. 61-69, 2010.
[8] L. Sun, and Y. Wang, "Application of structure-changed fuzzy neural network based on clonal algorithm in AGC system", Research on Iron and Steel, vol. 36, no. 4, pp. 37-40, 2008.

[9] M. and Afshari, and H. Sajedi, "A novel artificial immune algorithm for solving the job shop scheduling problem," International Journal of Computer Applications, vol. 48, no. 14, pp. 46-53, 2012.

Received: June 10, 2015

Revised: July 29, 2015

Accepted: August 15, 2015

(C) Kai et al.; Licensee Bentham Open.

This is an open access article licensed under the terms of the Creative Commons Attribution Non-Commercial License (http://creativecommons.org/licenses/by-nc/4.0/) which permits unrestricted, non-commercial use, distribution and reproduction in any medium, provided the work is properly cited. 